\title{
Effects of tobacco-peanut relay intercropping on soil bacteria community structure
}

\author{
Lin Gao $^{1} \cdot$ Xin-min Liu ${ }^{1} \cdot$ Yong-mei Du ${ }^{1} \cdot$ Hao Zong ${ }^{2} \cdot$ Guo-ming Shen ${ }^{1}$
}

Received: 4 July 2019 / Accepted: 13 November 2019 / Published online: 2 December 2019

(C) The Author(s) 2019

\begin{abstract}
Purpose A reasonable cultivation pattern is beneficial to maintain soil microbial activity and optimize the structure of the soil microbial community. To determine the effect of tobacco-peanut (Nicotiana tabacum-Arachis hypogaea) relay intercropping on the microbial community structure in soil, we compared the effects of relay intercropping and continuous cropping on the soil bacteria community structure.

Methods We collected soil samples from three different cropping patterns and analyzed microbial community structure and diversity using high-throughput sequencing technology.

Result The number of operational taxonomic units (OTU) for bacterial species in the soil was maximal under continuous peanut cropping. At the phylum level, the main bacteria identified in soil were Proteobacteria, Actinobacteria, and Acidobacteria, which accounted for approximately $70 \%$ of the total. The proportions of Actinobacteria and Firmicutes increased, whereas the proportion of Proteobacteria decreased in soil with tobacco-peanut relay intercropping. Moreover, the proportions of Firmicutes and Proteobacteria among the soil bacteria further shifted over time with tobacco-peanut relay intercropping. At the genus level, the proportions of Bacillus and Lactococcus increased in soil with tobacco-peanut relay intercropping.

Conclusion The community structure of soil bacteria differed considerably with tobacco-peanut relay intercropping from that detected under peanut continuous cropping, and the proportions of beneficial bacteria (the phyla Actinobacteria and Firmicutes, and the genera Bacillus and Lactococcus) increased while the proportion of potentially pathogenic bacteria (the genera Variibacter and Burkholderia) decreased. These results provide a basis for adopting tobacco-peanut relay intercropping to improve soil ecology and microorganisms, while making better use of limited cultivable land.
\end{abstract}

Keywords Tobacco $\cdot$ Peanut $\cdot$ Relay intercropping $\cdot$ High-throughput sequencing technology $\cdot$ Bacteria community structure

\section{Introduction}

Peanut is one of the most important oilseeds and cash crops worldwide as the primary source of vegetable oil and protein in developing countries. China is the largest peanut-producing and peanut-consuming country in the world (Yang and Zheng 2016; Ren et al. 2014). In recent years, with the continuous decline of cultivated land area, the competition between grain

\section{Guo-ming Shen}

gaolin@caas.cn

1 Tobacco Research Institute of Chinese Academy of Agricultural Sciences, Qingdao 266101, Shandong, China

2 Linyi Branch of Shandong Provincial Tobacco Company, Linyi 276001, Shandong, China and oil crops has become increasingly prominent in China. At the same time, the continuous cropping of peanut has generated several problems such as a lower yield, poor quality, and the emergence of diseases, which have seriously restricted development of the peanut industry (Li et al. 2012; Wang and Chen 2005). Intercropping, the historical practice of growing two or more crops together in the same field is one of the main planting methods of ecological and sustainable agriculture. Intercropping is a strategy for growing more crops in limited land, taking advantage of the crops' natural growth patterns to effectively match crops that can grow together so as to maximize the cultivation space. Intercropping is an essential practice to obtain high production while maintaining the quality of growing crops (Sullivan 2003; Seran and Brintha 2010; Alemayehu et al. 2017). In China, farmers have adopted intercropping systems for several years to increase production and decrease erosion. Peanut is suitable for 
intercropping with several types of crops in many ways. In particular, intercropping peanut with sugarcane or other annual crops such as maize, soybean, or upland rice is highly profitable (Feng et al. 2016; Li et al. 2016; Dana et al. 2017). Intercropping of peanut and flue-cured tobacco is a unique cropping system in tobacco-growing areas of China. This method can have a substantial benefit toward alleviating the continuous cropping problem and thus improve the utilization efficiency of the land.

Flue-cured tobacco is a special type of cash crop, and its root exudates have large effects on soil characteristics ( $\mathrm{Li}$ et al. 2017). Flue-cured tobacco root exudates can increase the content of available nutrients in soil, significantly affect the activities of phytases, catalase, and polyphenol oxidase (Lung et al. 2008; Yang et al. 2019). In addition, organic acids in fluecured tobacco root exudates can participate in plant growth regulation as chemical attractants to stimulate microbial growth (Faure et al. 2009; Fang et al. 2016). Soil microorganisms are an important component of the soil, playing an essential role in the processes of nutrient transformation and humus formation in soil. The soil microbial diversity and changes reflect the soil quality to some extent (Muhammad et al. 2018; Schloter et al. 2018). Continuous cropping can readily decrease the soil microbial activity and disturb the microbial population structure, which is the main contributor to the problems associated with continuous cropping ( $\mathrm{Li}$ et al. 2014). Indeed, studies have shown that a reasonable cultivation pattern is beneficial to maintain soil microbial activity and optimize the structure of the soil microbial community, thereby improving the soil ecological environment and overcoming the continuous cropping problem (Xiao et al. 2018; Tian et al. 2019). What are the effects of tobacco-peanut relay intercropping on soil microbial populations? However, there have been few reports on the effect of relay intercropping peanut with flue-cured tobacco on the soil microbial community. Therefore, the aim of the present study was to determine the effect of tobacco-peanut relay intercropping on the soil bacteria community structure using high-throughput sequencing technology. These results could provide a theoretical basis for the application and optimization of the intercropping pattern of peanut and flue-cured tobacco.

\section{Material and methods}

\section{Site description}

The experiment was conducted in the town of Xianggou, Junan County, Linyi City, Shandong Province, China, in 2016. The experimental region is part of the continental monsoon climate zone and the soil type is aquic brown soil according to FAO classification (Singer 2008) with the following properties: $\mathrm{pH}, 7.15$ (soil-to-water ratio of 1:2.5); organic matter, $10.7 \mathrm{~g} \mathrm{~kg}^{-1}$; alkali-hydrolyzable nitrogen, $70.40 \mathrm{mg}$ $\mathrm{kg}^{-1}$; available phosphorus, $43.21 \mathrm{mg} \mathrm{kg}^{-1}$; and available potassium, $116.7 \mathrm{mg} \mathrm{kg}^{-1}$.

\section{Soil sampling}

The sampling sites were located in the same ecological area, and three different cropping patterns were evaluated and compared: peanut continuous cropping $(\mathrm{J} 1)$, tobacco-peanut intercropping for 2 years (J2), and tobacco-peanut intercropping for 4 years (J3). For each cropping patterns, three farmer's fields were randomly selected as three replicates. For each of the sampled fields, the cultivation and management measures were obtained in accordance with the local technical regulations for flue-cured tobacco and peanut production. The variety of flue-cured tobacco and peanut planted in the fields was Yunyan 87 and Fenghua 1 respectively.

Soil samples were collected on September 15, 2016 when the peanuts were at the maturity stage. The sampling time was sunny morning, the air temperature was 25 degrees, and the relative humidity was $65 \%$. Sampling was performed randomly using multi-point mixing. Five sample points were selected from each field using an S-shaped distribution. Each soil sample was taken from peanut rhizosphere in each field by a fivespot-sampling method. After the peanut was gently pulled out, the whole root system was beaten to make the loose soil fall off completely. Then, the soil attached to the root surface was collected by the sterile brush as the rhizosphere soil. The composite soil samples were immediately transported to the laboratory in plastic bags via a portable refrigerator. Plant residues and other organic debris were carefully removed using forceps. The samples were kept moist and stored at $-40{ }^{\circ} \mathrm{C}$ for more than 1 month until measurement of the microbial community structure.

\section{Extraction of genomic DNA}

The total genomic DNA of soil samples was extracted using the Mo Bio microbial DNA extraction kit (Power Soil ${ }^{\mathrm{TM}}$ DNA Isolation Kit, Mo Bio, USA) according to the manufacturer instructions. The integrity of the DNA was determined by $1 \%$ agarose gel electrophoresis, while the purity and concentration of the DNA were assessed using a nanodrop microvolume spectrophotometer (Thermo Fisher Scientific).

\section{Amplicon generation}

The following primers were used to generate amplicons: $16 \mathrm{~S}$ V4, 515F(5'-GTGCCAGCMGCCGCGGTAA-3')-806R(5'GGACTACNVGGGTWTCTAA-3'). The 16S rRNA genes were amplified using specific primers with the required barcodes. All polymerase chain reactions (PCRs) were conducted in $30-\mu \mathrm{L}$ volumes with $15 \mu \mathrm{L}$ of Phusion ${ }^{\circledR}$ High- 
Fidelity PCR Master Mix (New England Biolabs), $0.2 \mu \mathrm{M}$ of forward and reverse primers, and approximately $10 \mathrm{ng}$ of template DNA. Thermal cycling conditions were as follows: initial denaturation at $98^{\circ} \mathrm{C}$ for $1 \mathrm{~min} ; 30$ cycles of denaturation at $98^{\circ} \mathrm{C}$ for $10 \mathrm{~s}$, annealing at $50^{\circ} \mathrm{C}$ for $30 \mathrm{~s}$, and elongation at $72{ }^{\circ} \mathrm{C}$ for $60 \mathrm{~s}$; and $72{ }^{\circ} \mathrm{C}$ for $5 \mathrm{~min}$.

For PCR product quantification and qualification, the same volume of $1 \times$ loading buffer (containing SYBR green) was mixed with the PCR products, and electrophoresis was conducted on a $2 \%$ agarose gel for detection. Samples exhibiting a bright main strip between 400 and $450 \mathrm{bp}$ were selected for further experiments. The PCR products were mixed in equidensity ratios, and the mixtures were purified using a GeneJET Gel Extraction Kit (Thermo Scientific).

\section{Library preparation and sequencing}

Sequencing libraries were generated using a NEB Next ${ }^{\circledR}$ Ultra $^{\mathrm{TM}}$ DNA Library Prep Kit for Illumina (NEB, USA) following the manufacturer's recommendations, and index codes were added. Library quality was assessed using a Qubit 2.0 fluorometer (Thermo Scientific) and an Agilent Bioanalyzer 2100 system. Libraries were then sequenced on an IlluminaMiSeq platform, and 250-300-bp paired-end reads were generated.

\section{Data analysis}

Firstly, the original sequencing data was spliced and filtered to obtain effective data. Then, operational taxonomic unit (OTU) clustering and species classification analyses were performed. OTUs and species annotations were then combined to retrieve the results of the basic analysis and the taxonomic pedigrees for each sample. In order to compute alpha diversity, we rarify the OTU table and calculate three metrics: Chaol estimates the species abundance, observed species estimates the amount of unique OTUs found in each sample, and Shannon index. Cluster analysis was preceded by principal component analysis (PCA), which was applied to reduce the dimension of the original variables using the QIIME software package.

\section{Results}

\section{Operational taxonomic unit identification}

Site J3 showed the highest total number of tags for soil bacteria sequences among the three sites (Table 1). However, the highest number of OTUs associated with bacterial species was found in J1 (Fig. 1). There were 812 OTUs in common in the soils of different cropping patterns, and $\mathrm{J} 1$ showed the highest number of unique OTUs.
Table 1 Number of tags and operational taxonomic units (OTUs) of bacterial communities

\begin{tabular}{lll}
\hline Treatment & Total tags & OTUs \\
\hline J1 & $13,410.67 \mathrm{~b}$ & $955.67 \mathrm{a}$ \\
J2 & $14,749.33 \mathrm{~b}$ & $861.33 \mathrm{~b}$ \\
J3 & $17,263.00 \mathrm{a}$ & $820.00 \mathrm{~b}$ \\
\hline
\end{tabular}

Different lowercase letters in the same column represent significant difference at $P<0.05$

\section{Species abundance}

The community structure of soil bacteria (at the phylum level) varied among the cropping patterns. Overall, the predominant bacteria in the soils were Proteobacteria, followed by Actinobacteria and Acidobacteria, accounting for approximately $70 \%$ of the total. The proportions of Actinobacteria and Firmicutes in the soil increased as a result of tobaccopeanut relay intercropping, whereas the proportion of Proteobacteria in soil decreased. After 4 years of tobaccopeanut relay intercropping, the proportion of Firmicutes in soil further increased and that of Proteobacteria decreased more obviously (Table 2).

At the genus level, tobacco-peanut relay intercropping did not increase the abundance of bacteria in soil, and their proportions were lower those in the continuous cropping soil. The relative abundances of Variibacter and Burkholderia decreased in soil bacteria with tobacco-peanut intercropping. However, the relative abundances of Bacillus and Lactococcus in soil significantly increased after tobaccopeanut relay intercropping (Table 3).

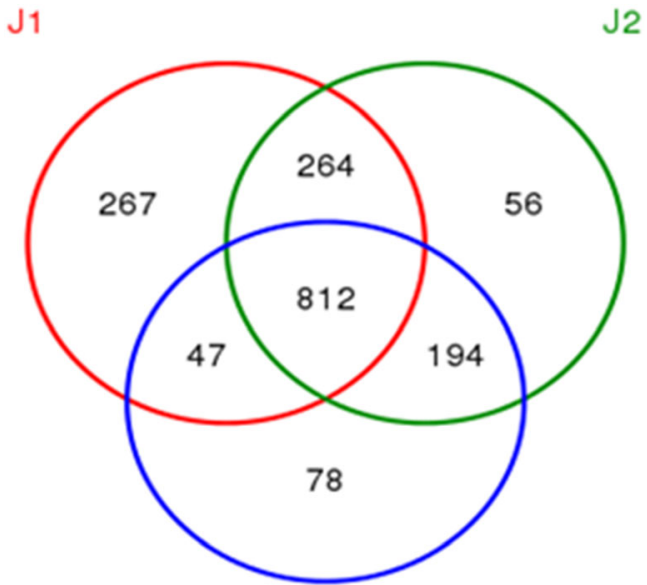

J3

Fig. 1 Venn classification map of soil bacterial operational taxonomic units in the three study sites. J1, peanut continuous cropping; J2, peanut-tobacco intercropping for 2 years; J3, peanut-tobacco intercropping for 4 years 
Table 2 Relative abundances of the soil bacterial community at the phylum level under three peanut cropping patterns

\begin{tabular}{lccc}
\hline Species (phylum level) & J1 (\%) & J2 (\%) & J3 (\%) \\
\hline Proteobacteria & $42.93 \mathrm{a}$ & $31.88 \mathrm{~b}$ & $25.41 \mathrm{~b}$ \\
Actinobacteria & $14.91 \mathrm{~b}$ & $24.29 \mathrm{a}$ & $23.02 \mathrm{a}$ \\
Acidobacteria & $15.34 \mathrm{a}$ & $17.15 \mathrm{a}$ & $16.52 \mathrm{a}$ \\
Chloroflexi & $9.21 \mathrm{a}$ & $10.88 \mathrm{a}$ & $10.66 \mathrm{a}$ \\
Firmicutes & $5.32 \mathrm{c}$ & $8.42 \mathrm{~b}$ & $15.69 \mathrm{a}$ \\
Gemmatimonadetes & $6.57 \mathrm{a}$ & $3.53 \mathrm{~b}$ & $2.78 \mathrm{~b}$ \\
Nitrospirae & $1.55 \mathrm{a}$ & $0.60 \mathrm{a}$ & $0.65 \mathrm{a}$ \\
Bacteroidetes & $1.53 \mathrm{a}$ & $0.63 \mathrm{~b}$ & $0.46 \mathrm{~b}$ \\
Saccharibacteria & $0.29 \mathrm{c}$ & $0.63 \mathrm{~b}$ & $1.62 \mathrm{a}$ \\
Verrucomicrobia & $0.92 \mathrm{a}$ & $0.52 \mathrm{a}$ & $0.74 \mathrm{a}$ \\
Other & $1.43 \mathrm{a}$ & $1.48 \mathrm{a}$ & $2.44 \mathrm{a}$ \\
\hline
\end{tabular}

Different lowercase letters in the same line represent significant difference at $P<0.05$

\section{Alpha diversity}

Alpha diversity is a comprehensive index reflecting species richness and evenness. Under a 97\% similarity level cutoff, the OTU $\alpha$-diversity of each sample was calculated, and the Shannon and Chaol indices were used to analyze the soil microbial diversity. The Chao1 and Shannon indices of soil bacteria differed among the cropping patterns, with the lowest diversity detected under tobacco-peanut relay intercropping for 4 years (Table 4). Both the richness and diversity of soil bacteria were reduced by tobacco-peanut relay intercropping.

\section{Principal components analysis}

The PCA plot clearly separated soil samples subjected to peanut continuous cropping and tobacco-peanut relay intercropping. There was much greater variation among the

Table 3 Relative abundances of the soil bacterial community at the genus level under three peanut cropping patterns

\begin{tabular}{llll}
\hline Species (genus level) & $\mathrm{J} 1(\%)$ & $\mathrm{J} 2(\%)$ & $\mathrm{J} 3(\%)$ \\
\hline Unidentified & $59.88 \mathrm{a}$ & $52.12 \mathrm{a}$ & $50.49 \mathrm{a}$ \\
Bacillus & $2.05 \mathrm{~b}$ & $3.29 \mathrm{~b}$ & $6.49 \mathrm{a}$ \\
Sphingomonas & $2.30 \mathrm{a}$ & $2.54 \mathrm{a}$ & $1.90 \mathrm{a}$ \\
Lactococcus & $0.03 \mathrm{c}$ & $1.67 \mathrm{~b}$ & $4.45 \mathrm{a}$ \\
Bradyrhizobium & $1.35 \mathrm{~b}$ & $2.22 \mathrm{a}$ & $1.93 \mathrm{a}$ \\
Gemmatimonas & $2.12 \mathrm{a}$ & $1.29 \mathrm{a}$ & $1.01 \mathrm{a}$ \\
Rhizomicrobium & $1.54 \mathrm{a}$ & $1.23 \mathrm{a}$ & $1.42 \mathrm{a}$ \\
Acidibacter & $1.33 \mathrm{a}$ & $1.44 \mathrm{a}$ & $0.72 \mathrm{a}$ \\
Variibacter & $1.11 \mathrm{a}$ & $0.57 \mathrm{~b}$ & $0.25 \mathrm{~b}$ \\
Burkholderia & $1.39 \mathrm{a}$ & $1.10 \mathrm{a}$ & $0.62 \mathrm{~b}$ \\
Other & $27.90 \mathrm{a}$ & $32.23 \mathrm{a}$ & $30.24 \mathrm{a}$ \\
\hline
\end{tabular}

Different lowercase letters in the same line represent significant difference at $P<0.05$
Table 4 Chao1 and Shannon indices of soil bacteria under different peanut cropping systems

\begin{tabular}{lll}
\hline Treatment & Shannon index & Chao1 index \\
\hline J1 & $8.36 \mathrm{a}$ & $1093.11 \mathrm{a}$ \\
$\mathrm{J} 2$ & $7.84 \mathrm{ab}$ & $963.17 \mathrm{~b}$ \\
$\mathrm{~J} 3$ & $7.36 \mathrm{~b}$ & $892.46 \mathrm{~b}$ \\
\hline
\end{tabular}

Different lowercase letters in the same column represent significant difference at $P<0.05$

bacterial species under the tobacco-peanut relay intercropping pattern than under continuous cropping. However, the soil bacteria species were similar overall under the tobaccopeanut relay intercropping for 2 and 4 years (Fig. 2).

\section{Discussion}

Different cropping patterns can affect soil environmental conditions, especially the intercropping patterns can change soil temperature, moisture, and light conditions due to the interaction between crops, and thus affect soil properties to consequently influence the activities and community structure of the soil microorganisms (Berg and Smalla 2009; Hayat et al. 2010; Jangid et al. 2008). Intercropping has been shown to be superior to rotation practice in terms of crop productivity, even after only two cropping seasons. The benefits resulting from intercropping should be tightly linked to changes in soil microbial functionalities, and the changes in crop communities could stimulate specific functional traits of soil microbial communities. Indeed, intercropping has a substantial impact on soil microbial activity and community structure. For example, relay intercropping of garlic with cucumbers was shown to alter the soil bacterial community structure, increase the

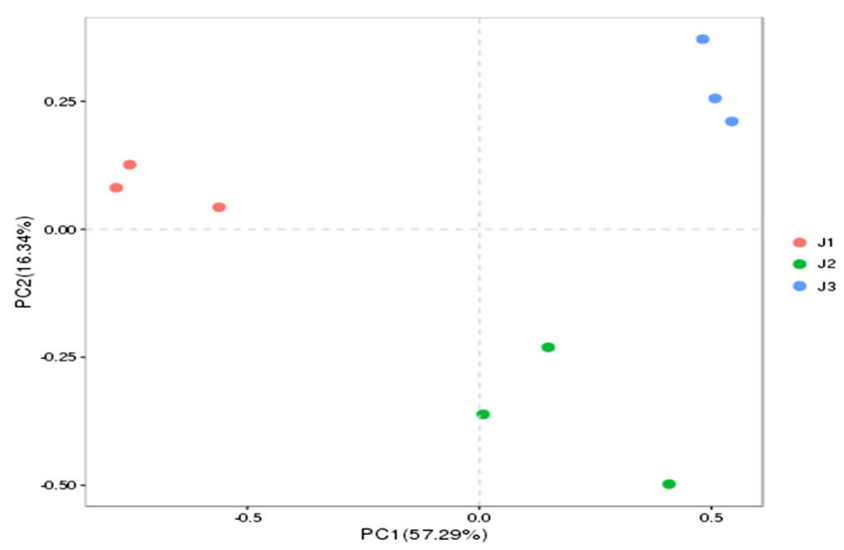

Fig. 2 Principal component analysis of the soil bacteria community. J1, peanut continuous cropping; J2, peanut-tobacco intercropping for 2 years; J3, peanut-tobacco intercropping for 4 years 
bacterial diversity indices, and enrich the dominant bacterial populations in the soil (Du et al. 2017). Similarly, legume crop rotations have been reported to significantly affect the microbial community structure and increase microbial diversity. Similar results have been obtained in intercropping experiments in which the bacterial biomass and activity differed from those detected in mono-cropping systems (Duchene et al. 2017). However, in the present study, both the richness and diversity of soil bacteria showed a downward trend in the tobacco-peanut relay intercropping system (Table 4). Different crop root exudates that accumulate in the soil change the soil microbial nutrient conditions, which could affect the species distribution, abundance, and diversity of microorganisms in the soil. Root exudation plays a key role in ensuring crop-microorganism interactions and significantly shapes the structure of microbial communities (Badri and Vivanco 2009; Cipollini et al. 2012). Tobacco is a special economic crop, which secretes a variety of root exudates, many of which have been identified to exhibit strong allelopathy. Indeed, tobacco planting has a great influence on soil microorganisms (Fang et al. 2016; Liu et al. 2015), and intercropping tobacco with other crops can alter microbial activity and community structure in the soil. In addition, the proportions of the phyla Bacteroidetes and Acidobacterium were significantly affected among soil bacteria with figwort (Scrophularia ningpoensis)tobacco intercropping (Zhang et al. 2017). In the present study, we found that tobacco-peanut intercropping increased the proportions of Actinobacteria and Firmicutes in the soil, but decreased the proportion of Proteobacteria (Table 2). Proteobacteria includes some pathogenic bacteria, and the genera Variibacter were detected from soil in this study. The relative abundances of Variibacter decreased in soil bacteria with tobacco-peanut intercropping (Table 3). In addition, in this study, the genera Burkholderia were also detected from soil and its relative abundances decreased with tobaccopeanut intercropping. Burkholderia is not always pathogenic, some of them are plant growth-promoting rhizobacteria, which species changes led to the reduction of the relative abundance of Burkholderia, and further analysis is needed in the future.

The community composition and quantitative changes of microorganisms in the rhizosphere soil greatly contribute to crop diseases, especially soil-borne diseases. Previous studies demonstrated that intercropping can mitigate crop diseases, which is strongly associated with the resulting changes in the soil microbial community structure (Fernández-Aparicio et al. 2010; Abdel-Monaim and Abo-Elyousr 2012; Enikuomehin et al. 2010 ). In a reasonable intercropping system, the roots of crops are interlaced with each other, producing a variety of rhizosphere exudates to exert a more obvious rhizosphere effect. Thus, intercropping can optimize the structure of the soil microbial community and improve soil health
(Boudreau 2013). Indeed, our study showed that the relative abundances of Bacillus and Lactococcus significantly increased in soil with tobacco-peanut intercropping (Table 3). Bacillus spp. often produces spores with special resistance to adverse environmental conditions, many of which are plant growth-promoting rhizobacteria. Furthermore, Bacillus spp. can secrete a variety of bioactive substances such as antibacterial proteins and growth hormones, which can improve crop resistance, inhibit disease occurrence, and promote normal growth and development of crops (Anusha et al. 2019; Viviana and César 2018). In addition, Bradyrhizobium was also detected from tobacco-peanut intercropping soils(Table 3). Bradyrhizobium can fix atmospheric nitrogen and supply it to host plants for absorption and utilization. The relative abundance of Bradyrhizobium in soil bacteria increased with tobacco-peanut intercropping, which was beneficial to the optimum utilization of nitrogen. Therefore, tobacco-peanut intercropping could improve the number of beneficial bacteria in soil and decrease the number of potential pathogens. Overall, these results indicate that adoption of a reasonable intercropping technique of flue-cured tobacco and peanut has positive effects on preventing the problems associated with the continuous cropping of peanut.

\section{Conclusions}

This study shows that the community structure of soil bacteria was markedly influenced by tobacco-peanut relay intercropping, with evident shifts in the composition and abundances compared with those from peanut continuous cropping soil. In particular, the proportions of Actinobacteria and Firmicutes increased in the soil under tobacco-peanut relay intercropping, whereas the proportion of Proteobacteria decreased. Further analysis at the genus level showed the relative abundances of Varibacter and Burkholderia decreased in soil bacteria with tobacco-peanut intercropping, whereas the relative abundances of Bacillus and Lactococcus significantly increased. Moreover, the richness and diversity of soil bacteria reduced with tobaccopeanut relay intercropping compared with continuous cropping.

Funding information This work was financially supported by the China National Tobacco Corporation (Project no. 110201202014).

\section{Compliance with ethical standards}

Conflict of interest The authors declare that they have no conflict of interest. 
Research involving human participants and/or animals This article does not contain any studies with human participants or animals performed by any of the authors.

Informed consent Informed consent is not applicable in this study.

Open Access This article is distributed under the terms of the Creative Commons Attribution 4.0 International License (http:// creativecommons.org/licenses/by/4.0/), which permits unrestricted use, distribution, and reproduction in any medium, provided you give appropriate credit to the original author(s) and the source, provide a link to the Creative Commons license, and indicate if changes were made.

\section{References}

Abdel-Monaim MF, Abo-Elyousr KAM (2012) Effect of preceding and intercropping crops on suppression of lentil damping-off and root rot disease in New Valley, Egypt. Crop Prot 32:41-46

Alemayehu A, Tamado T, Nigussie D et al (2017) Maize-common bean intercropping to optimize maize-based crop production. J Agric Sci 155:1124-1136

Anusha BG, Gopalakrishnan S, Naik MK et al (2019) Evaluation of Streptomyces spp. and Bacillus spp. for biocontrol of Fusarium wilt in chickpea (Cicer arietinum L.). Arch Phytopathol Plant Protect 52(5):417-442

Badri DV, Vivanco JM (2009) Regulation and function of root exudates. Plant Cell Environ 32:666-681

Berg G, Smalla K (2009) Plant species and soil type cooperatively shape the structure and function of microbial communities in the rhizosphere. FEMS Microbiol Ecol 68:1-13

Boudreau MA (2013) Diseases in intercropping systems. Annu Rev Phytopathol 51:499-519

Cipollini D, Rigsby CM, Barto EK (2012) Microbes as targets and mediators of allelopathy in plants. J Chem Ecol 38:714-727

Dana A, Ganjali HR, Mehraban A (2017) The evaluation of yield and yield component of corn (Zea mays) and peanut (Arachis hypogaea) affect by intercropping and manure. Int J Agr Biosci 6:64-67

Du LT, Huang BJ, Du NS et al (2017) Effects of garlic/cucumber relay intercropping on soil enzyme activities and the microbial environment in continuous cropping. Hortscience 52(1):78-84

Duchene O, Vian JF, Celette F (2017) Intercropping with legume for agroecological cropping systems: complementarity and facilitation processes and the importance of soil microorganisms. A review. Agric Ecosyst Environ 240:148-161

Enikuomehin OA, Aduwo AM, Olowe VIO et al (2010) Incidence and severity of foliar diseases of sesame (Sesamum indicum L.) intercropped with maize (Zea mays L.). Arch Phytopathol Plant Protect 43:972-986

Fang YT, Zhang LM, Jiao YG et al (2016) Tobacco rotated with rapeseed for soil-borne phytophthora pathogen biocontrol: mediated by rapeseed root exudates. Front Microbiol 7:894

Faure D, Vereecke D, Leveau JH (2009) Molecular communication in the rhizosphere. Plant Soil 321:279-303

Feng LS, Sun ZX, Zheng MZ et al (2016) Productivity enhancement and water use efficiency of peanut-millet intercropping. Pak J Bot 48(4): $1459-1466$

Fernández-Aparicio M, Amri M, Kharrat M, Rubiales D (2010) Intercropping reduces Mycosphaerella pinodes severity and delays upward progress on the pea plant. Crop Prot 29(7):744-750
Hayat R, Ali S, Amara U et al (2010) Soil beneficial bacteria and their role in plant growth promotion: a review. Ann Microbiol 60:579598

Jangid K, Williams MA, Franzluebbers AJ et al (2008) Relative impacts of land-use, management intensity and fertilization upon soil microbial community structure in agricultural systems. Soil Biol Biochem 40(11):2843-2853

Li PD, Dai CC, Wang XX et al (2012) Variation of soil enzyme activities and microbial community structure in peanut monocropping system in subtropical China. Afr J Agric Res 7(12):1870-1879

Li XG, Ding CF, Hua K et al (2014) Soil sickness of peanuts is attributable to modifications in soil microbes induced by peanut root exudates rather than to direct allelopathy. Soil Biol Biochem 78:149 159

Li QS, Wu LK, Chen J et al (2016) Biochemical and microbial properties of rhizospheres under maize/peanut intercropping. J Integr Agric 15(1):101-110

Li SL, Xu C, Wang J et al (2017) Cinnamic, myristic and fumaric acids in tobacco root exudates induce the infection of plants by Ralstonia solanacearum. Plant Soil 412(2):381-395

Liu YX, Li X, Cai K et al (2015) Identification of benzoic acid and 3phenylpropanoic acid in tobacco root exudates and their role in the growth of rhizosphere microorganisms. Appl Soil Ecol 93:78-87

Lung SC, Leung A, Kuang R et al (2008) Phytase activity in tobacco (Nicotiana tabacum) root exudates is exhibited by a purple acid phosphatase. Phytochemistry 69(2):365-373

Muhammad A, Sidra M, Afifa M et al (2018) Paddy soil microbial diversity and enzymatic activity in relation to pollution. Env Poll Paddy Soils 53:139-149

Ren X, Jiang H, Yan Z et al (2014) Genetic diversity and population structure of the major peanut (Arachis hypogaea L.) cultivars grown in China by SSR markers. PLoS One 9(2):e88091

Schloter M, Nannipieri P, Sørensen SJ et al (2018) Microbial indicators for soil quality. Biol Fertil Soils 54(1):1-10

Seran TH, Brintha I (2010) Review on maize based intercropping. J Agron 9:135-145

Singer A (2008) Classification of Soils: FAO[M]. Springer, Netherlands

Sullivan P (2003) Intercropping principles and production practices. University of Arkansas, Fayetteville

Tian XL, Wang CB, Bao XG et al (2019) Crop diversity facilitates soil aggregation in relation to soil microbial community composition driven by intercropping. Plant Soil 436(1):173-192

Viviana YM, César EF (2018) Efficacy of bacillus spp. to biocontrol of anthracnose and enhance plant growth on Andean lupin seeds by lipopeptide production. Biol Control 122:67-75

Wang MZ, Chen XN (2005) Obstacle and countermeasure of sustainable high yield for peanut in low-hilly red soil region. J Peanut Sci 34: $17-22$

Xiao L, Huang YM, Zeng QC et al (2018) Soil enzyme activities and microbial biomass response to crop types on the terraces of the Loess Plateau, China. J Soils Sediments 18(5):1971-1980

Yang TK, Zheng Y (2016) State and trends of oil crops production in China. Oilseeds fats Crops Lipids 23(6):D603

Yang YF, Yang ZX, Yu SZ et al (2019) Organic acids exuded from roots increase the available potassium content in the rhizosphere soil: a rhizobag experiment in nicotiana tabacum. Hortscience 54(1):23-27

Zhang DY, Wang J, Yang SP (2017) Influence of Scrophularia ningpoensis-tobacco intercropping on bacterial community structure in soil. Acta Pratacul Sin 26(6):120-130

Publisher's note Springer Nature remains neutral with regard to jurisdictional claims in published maps and institutional affiliations. 